\title{
Necrosis or Flap Loss After Deep Inferior Epigastric Perforator Reconstruction: Impact of Perforators and Recipient Vessels
}

\author{
Johanna S. Palve, MD, PhD ${ }^{1}$ Tiina H. Luukkaala, $\mathrm{MSc}^{2} \quad$ Minna T. Kääriäinen, MD, $\mathrm{PhD}^{1}$ \\ ${ }^{1}$ Department of Plastic Surgery, Tampere University, Faculty of \\ Address for correspondence Johanna Palve, MD, PhD, Tampere \\ Medicine and Health Technology and Tampere University Hospital, \\ Tampere, Finland \\ 2 Research, Development and Innovation Center, Tampere University \\ University Hospital, Department of Plastic Surgery, Elämänaukio 2, \\ 33521 Tampere, Finland (e-mail: johanna.palve@pshp.fi).
} Hospital and Health Sciences, Faculty of Social Sciences, Tampere University, Tampere, Finland

J Reconstr Microsurg Open 2021;6:e20-e27.

\author{
Abstract \\ Keywords \\ - deep inferior \\ epigastric perforator \\ - perforator \\ - recipient vessel \\ - flap necrosis
}

Background The aim of this study is to analyze the impact of the number and location of perforators harvested and the recipient vessels used on deep inferior epigastric perforator (DIEP) flap survival and the occurrence of flap necrosis requiring reoperation.

Patients and Methods Four hundred and seventy-one DIEP flap reconstructions, performed between January 2008 and December 2019, were retrospectively analyzed. Results Flap necrosis requiring re-operation was observed in 40 (9\%) of flaps and total flap loss rate was $1 \%(n=6)$. No significant differences were observed between internal mammary vessels (IMV, $n=287,61 \%$ ) and thoracodorsal vessels (TDV, $n=184,39 \%$ ) regarding postoperative re-anastomosis $(p=0.529)$ or flap survival $(p=0.646)$. Intraoperative conversion from IMV to TDV was performed on 64 (14\%) patients. TDV were more commonly associated with problems in preparation of the vessels than IMV $(p<0.001)$. Second vein anastomosis was performed on $18(4 \%)$ patients. In total, 81 flaps (17\%) had one perforator, 165 (35\%) had two, 218 (46\%) had three to five, and 7 (2\%) had more than five perforators. Flaps with three to five perforators were more commonly associated with flap necrosis $(p<0.001)$ than flaps with one or two perforators. Independent factors associated with necrosis were body mass index $(\mathrm{BMI})>30$ (odds ratio [OR]: 2.28; 95\% confidence interval: 1.06-4.91, $p=0.035$ ) and perforator/s located on the lateral row (OR: 3.08, 95\% Cl 1.29-7.33, $p=0.011$ ). Conclusion We conclude that the occurrence of flap necrosis requiring re-operation may be higher in DIEP flaps with more than two perforators or perforator/s located on the lateral row and in obese patients. Neither the recipient vessels used nor the number of perforators harvested had any impact on the flap survival rate. received

October 20, 2020

accepted

January 11, 2021
DOI https://doi.org/ $10.1055 / \mathrm{s}-0041-1729638$. ISSN $2377-0813$.

\footnotetext{
(C) 2021. The Author(s).

This is an open access article published by Thieme under the terms of the Creative Commons Attribution-NonDerivative-NonCommercial-License, permitting copying and reproduction so long as the original work is given appropriate credit. Contents may not be used for commercial purposes, or adapted, remixed, transformed or built upon. (https://creativecommons.org/ licenses/by-nc-nd/4.0/) Thieme Medical Publishers, Inc., 333 Seventh Avenue, 18th Floor, New York, NY 10001, USA
} 
The deep inferior epigastric perforator (DIEP) flap has become the most popular option for autologous breast reconstruction. $^{1-3}$ The DIEP is a fasciocutaneous flap, the perfusion of which relies on tiny blood vessels known as perforators. ${ }^{4}$ These perforators are identified intraoperatively as they exit the rectus sheath and are then followed through the rectus muscle down to its pedicle. ${ }^{3}$ Meticulous perforator selection is important in providing adequate perfusion to the flap. ${ }^{5}$ Inadequate perfusion leads to skin and fat necrosis, which may require wound care and revisionary procedures. ${ }^{6}$ Many factors need to be considered when deciding which perforator/s are selected. These factors include the perforator diameter, intramuscular course, branching pattern, and perfusion zones. ${ }^{3}$

There is the concept of zonal perfusion in lower abdominal-based flaps, which has classically helped surgeons to estimate the tissue territory perfusion. ${ }^{7-10}$ The introduction of the "perforasome" concept revealed that the perfusion of DIEP flaps varies depending on the location, rows, and quality of the perforators. ${ }^{11}$ Preoperative imaging provides valuable information on the perforators and helps in the planning of the procedure, as there is great individual variation in vascular anatomy in the abdominal area. ${ }^{3,4}$ Several methods for preoperative planning have been applied. ${ }^{2,4,12}$ Of these, the mainstay is computed tomographic angiography (CTA). ${ }^{13}$ It allows for visualization of the deep inferior epigastric artery, its intramuscular course, and its branching pattern. $^{2}$

In the literature, conflicting results have been presented regarding the number of perforators and flap necrosis. Indeed, some studies have reported that flaps with a single ${ }^{14}$ or one to two ${ }^{15}$ perforators have a higher incidence of fat necrosis than those flaps with multiple perforators, whereas other $^{16}$ studies have reported that the incidence of fat necrosis increased with an increased number of perforators. Furthermore, some studies have debated whether perforator location has any impact on the incidence of fat necrosis. It has been further suggested that using perforators from the lateral row alone, or in addition to medial row perforators, can decrease fat necrosis. ${ }^{5}$ A large review study by Bhullar et al suggested that in the ideal flap, two to three medial or lateral row perforators of a substantial caliber would be harvested. ${ }^{3}$

In addition to meticulous perforator selection, the choice of recipient vessels is important for success. The choice of recipient vessels is always based on the surgeon's own experience. $^{17,18}$

The most commonly used recipient vessels are the thoracodorsal (TDV) and internal mammary vessels (IMV). 17,19,20 However, the use of the circumflex scapular pedicle and IMV perforators is also popular. ${ }^{17}$ Previous studies have reported that there are several advantages associated with the use of IMV compared with TDV. These advantages include better arterial inflow, shorter pedicle preparation, breast placement more medially, and the avoidance of axillary exploration. ${ }^{19}$ Indeed, the use of IMV as a first choice has been recommended since $1996 .{ }^{17}$ If IMV are not usable, the most common alternative recipient vessels used are TDV, lateral thoracic vessels, or serratus vessels. ${ }^{21}$ In patients who have undergone postmastectomy radiotherapy, however, the choice of recipient vessels may be more difficult because both the TDV and IMV will have been irradiated. In such cases, up to a $20 \%$ rate of IMV conversion has been reported. ${ }^{20}$ The use of axillary vessels as an alternate choice is also more complicated due to scarring after axillary clearance.

The aim of this study was to evaluate the impact of the harvested perforators and recipient vessels used on the outcome of DIEP flap reconstruction.

\section{Patients and Methods}

This retrospective study was conducted by using data from the breast reconstruction database at Tampere University Hospital (Finland). We identified all DIEP flap breast reconstruction operations performed from January 1, 2008 to December 31, 2019. Permission to access the clinical records of the patients for the study was obtained from the Science Center of Tampere University Hospital. By reviewing the clinical records, we ensured that there were no duplicates.

We collected data on patient characteristics, reconstruction indication, number and location of perforators, recipient vessels, and complications. Patient characteristics included age, body mass index (BMI), smoking status, comorbidities, a history of previous lower abdominal surgery/scars, and radiation therapy. Age was calculated in years on the day of the reconstruction. BMI was calculated in $\mathrm{kg} / \mathrm{m}^{2}$. Smoking status was dichotomized as "smoker" or "nonsmoker." "Nonsmokers" were patients who had never smoked, and "smokers" were patients who smoked or had stopped for a period of 4 weeks prior to reconstruction. Comorbidities were divided into diabetes, cardiovascular disease (CVD), and other (including asthma, chronic obstructive pulmonary disease [COPD] and hypothyreosis). Radiation therapy included radiotherapy before reconstruction. Reconstruction indications were delayed or immediate (including prophylactic procedures). The number and location (medial or lateral row) of the perforators included in the flap were recorded from clinical records. Information about recipient vessels and possible problems with them was also recorded. Retrograde IMV anastomoses were not performed.

The complications recorded were flap necrosis requiring re-operation and postoperative re-anastomosis. Flap necrosis included both symptomatic fat necrosis with wound dehiscence, but without skin necrosis, and fat necrosis with necrosis of the overlying skin. In flap debridement, all the necrotic tissue was removed and the defect size was measured after excision. In cases of total flap loss, the whole necrotic flap was removed. In this study, we did not have an information on asymptomatic fat necrosis because we did not routinely use ultrasound to identify possible small and asymptomatic necrosis. Further, we only recorded flap necrosis. Mastectomy skin loss was not included.

In our clinic, perforators for the flap were planned preoperatively according to CTA. The largest perforator was defined as the dominant perforator. The course of the perforator through the muscle to the pedicle was identified 
from CTA and any possible minor perforator/s running to the same pedicle were identified. Intraoperatively, the dominant perforator was first identified and after that the other perforators were also examined. If the dominant perforator was very large with a good pulse and the other perforator/s were only very small, the smaller perforator/s were sacrificed. If, on the other hand, there were smaller but still good caliber perforator/s, we made a temporary occlusion to the smaller perforator/s with clamps and then evaluated the perfusion of the flap. If the perfusion was good without smaller perforator/s, we sacrificed it, otherwise it was included. If there were two equally large perforators with a good pulse, both were included.

During the operation, zones 3 and 4 were excised in unilateral DIEPs to avoid flap necrosis. In bilateral flaps, no whole zones were removed. Usually, only the most lateral corner of the flap was excised regardless of the perforator row. The extension of the debridement was evaluated clinically. We did not use intraoperative imaging.

\section{Statistics}

The differences between vessels were analyzed using Pearson's Chi-square test, Fisher's exact test, or Mann-Whitney test. Univariable logistic regression and multivariable logistic regression analyses were performed to estimate odds ratios (ORs) and 95\% confidence intervals (CIs) to analyze the association between possible risk factors for complications. A $p$-value $<0.05$ was considered statistically significant. All analyses were conducted with IBM (SPSS statistics version 26, Armonk, NY: IBM Corp. Released 2019).

\section{Results}

The patient characteristics and DIEP flap outcomes are presented in - Table 1. The median age of patients was 53 years (range $=19-71$ ) and median BMI was 26.4 (interquartile range [IQR]: 24.0-29.0). Comorbidities were recorded in $28 \%(n=125)$ of patients, the most common comorbidity being cardiovascular disease. In total, 37 (8\%) patients were smokers. A history of previous lower abdominal operation (and scar/s) was recorded in 127 (27\%) patients. More than half $(57 \%, n=261)$ of the reconstruction areas had undergone previous radiation therapy. During the study period, a total of 471 DIEP reconstructions were performed on 446 patients. Most of the patients underwent delayed $(n=407,86 \%)$ and unilateral $(n=446,95 \%)$ reconstruction. The total flap loss rate was $1 \%$ (6 out of 471 flaps). Sixteen flaps (3\%) had an episode of postoperative venous and six flaps (1\%) had arterial thrombosis. Flap necrosis requiring re-operation was found in 40 (9\%) flaps. IMV were the most common recipient vessels $(n=287,61 \%)$ compared with TDV $(n=184,39 \%)$. A second vein anastomosis from the superficial vein was performed on $18(4 \%)$ patients. Most of these anastomoses $(n=13,72 \%)$ were performed on axillary vessels (thoracodorsal or serratus vena). Five anastomoses (28\%) were performed on a branch of the deep inferior epigastric vein. Retrograde IMV anastomoses were not performed.

The number and location of the harvested perforators are presented in - Table 2.The majority of the flaps had three to five $(n=218,46 \%)$ or two $(n=165,35 \%)$ perforators. In addition, $17 \%$ of the flaps $(n=81)$ had one perforator, which was more commonly located in the medial $(70 \%, n=57)$ than in the lateral $(30 \%, n=24)$ row. In flaps with three to five perforators, the perforators were more commonly located in both rows compared with flaps with two perforators (51 vs. $19 \%)$. Six flaps (7\%) experienced necrosis in the 1-perforator group, 12 (7\%) in the 2-perforator group, and $22(10 \%)$ in 3- to 5 -perforator group. This demonstrated a statistically significant difference in the rate of necrosis in those flaps with three to five perforators $(p<0.001)$.

Multivariable adjusted logistic regression was used to analyze the association between the incidence of flap necrosis and the variables of interest (-Table $\mathbf{3}$ ). In this analysis, the independent risk factors for flap necrosis requiring reoperation were BMI > 30 (OR: 2.29; 95\% CI: 1.06-4.94, 0.034) and location of the perforator/s in the lateral row (OR: 2.83; 95\% CI: $1.19-6.71 ; p=0.018)$. A previous operation was not an independent risk factor for flap necrosis.

An analysis according to the recipient vessels (IMV or TDV) was conducted ( - Table 4 ). No significant differences were observed among groups regarding postoperative re-anastomosis $(p=0.529)$ and flap survival after re-anastomosis $(p=0.646)$. Intraoperative conversion from IMV to TDV was performed on 64 (14\%) patients. There were no conversions from TDV to other vessels. Of those patients who underwent a conversion, 33 (52\%) had previously undergone radiation therapy and 38 (59\%) had a problem on the left side. In the preparation of vessels, problems were more common with TDV than with IMV ( 29 vs. $4 \%, p<0.001$ ). In our study cohort, 246 of 471 patients had axillary clearance due to metastasis. Patients who had intraoperative problems with TDV had axillary clearance in $58 \%$ of cases. Total flap losses occurred in four patients with IMV as recipient vessels compared with total flap losses in two patients with TDV as recipient vessels. Seven venous and three artery thrombosis were associated with TDV and nine venous and three artery thrombosis with IMV.

\section{Discussion}

In this study, we examined the DIEP flaps performed in our clinic to evaluate the impact of the recipient vessels used and the number and location of the harvested perforators on the outcome of the flaps. We had a $9 \%$ rate of flap necrosis requiring re-operation, which is comparable with earlier studies that reported an incidence of necrosis in the abdominal flaps of between 5 and $45 \%^{3,15}$ The evaluation of necrosis, however, varied between studies, ranging from firmer palpable areas to wound-healing complications, and necrotic skin and fat requiring re-operation. In this study, we counted only flap necrosis requiring re-operation. We did not have any information on asymptomatic fat necrosis because we did not routinely use ultrasound to identify 
Table 1 The overall patient characteristics and deep inferior epigastric perforator flap outcomes

\begin{tabular}{|c|c|}
\hline Patients $(n=446)$ & \\
\hline Age $(y)$, median (interquartile range; range) & $53(48-58 ; 19-71)$ \\
\hline BMI $\left(\mathrm{kg} / \mathrm{m}^{2}\right)$, median (interquartile range) & $26.4(24.0-29.0)$ \\
\hline $\mathrm{BMI}<30, n(\%)$ & $374(84)$ \\
\hline $\mathrm{BMI}>30, n(\%)$ & $72(16)$ \\
\hline Comorbidities, $n(\%)$ & $125(28)$ \\
\hline Diabetes & $12(3)$ \\
\hline Cardiovascular disease & $67(15)$ \\
\hline Asthma/COPD & $33(7)$ \\
\hline \multicolumn{2}{|l|}{ Smoking, $n(\%)$} \\
\hline No & $409(92)$ \\
\hline Yes & $37(8)$ \\
\hline Scars after previous lower abdominal operations & $127(27)$ \\
\hline Laparoscopic & $31(7)$ \\
\hline Horizontal or vertical scar & $45(10)$ \\
\hline Appedicectomy & $20(4)$ \\
\hline Multiple scars & $31(7)$ \\
\hline \multicolumn{2}{|l|}{ DIEP flaps $(n=471)$} \\
\hline \multicolumn{2}{|l|}{ Radiotherapy, $n$ (\%) } \\
\hline No & $198(43)$ \\
\hline Yes & $261(57)$ \\
\hline Bilateral, n (\%) & $25(5)$ \\
\hline Unilateral, $n(\%)$ & $446(95)$ \\
\hline Right, $n(\%)$ & $242(51)$ \\
\hline Left, $n(\%)$ & $229(49)$ \\
\hline \multicolumn{2}{|l|}{ Indication, $n(\%)$} \\
\hline Immediate & $64(14)$ \\
\hline Delayed & $407(86)$ \\
\hline \multicolumn{2}{|l|}{ Outcomes, $n(\%)$} \\
\hline Total flap loss & $6(1)$ \\
\hline Flap necrosis, $n(\%)$ & $40(9)$ \\
\hline Size in $\mathrm{cm}^{2}$, median (interquartile range, range) & $9(6-12,2-40)$ \\
\hline$<5 \mathrm{~cm}^{2}$ & $9(19)$ \\
\hline $5-9.9 \mathrm{~cm}^{2}$ & $14(33)$ \\
\hline $10-14.9 \mathrm{~cm}^{2}$ & $13(31)$ \\
\hline$\geq 15 \mathrm{~cm}^{2}$ & $7(17)$ \\
\hline Postoperative venous thrombosis & $16(3)$ \\
\hline Postoperative arteria thrombosis & $6(1)$ \\
\hline \multicolumn{2}{|l|}{ Recipient vessels, $n$ (\%) } \\
\hline IMV & $287(61)$ \\
\hline TDV & $184(39)$ \\
\hline Intraoperative conversion from IMV to TDV & $64(14)$ \\
\hline A second vein anastomosis needed & $18(4)$ \\
\hline
\end{tabular}

Abbreviations: BMI, body mass index; COPD, chronic obstructive pulmonary disease; DIEP, deep inferior epigastric perforator; IMV, internal mammary vessels; TDV, thoracodorsal vessel. 
Table 2 Number and location of perforators harvested in deep inferior epigastric perforator flaps $(n=471)$

\begin{tabular}{|c|c|c|c|c|c|c|c|c|}
\hline & \multicolumn{8}{|c|}{ Flaps grouped by number of perforators } \\
\hline & \multicolumn{2}{|l|}{1} & \multicolumn{2}{|l|}{2} & \multicolumn{2}{|l|}{$3-5$} & \multicolumn{2}{|c|}{$>5$} \\
\hline & $n$ & $(\%)$ & $n$ & $(\%)$ & $n$ & $(\%)$ & $n$ & $(\%)$ \\
\hline Number of flaps & 81 & $(17)$ & 165 & $(35)$ & 218 & $(46)$ & 7 & $(2)$ \\
\hline \multicolumn{9}{|l|}{ Location of perforators $^{a}$} \\
\hline Medial row & 57 & $(70)$ & 69 & $(42)$ & 31 & $(14)$ & 0 & \\
\hline Lateral row & 24 & $(30)$ & 64 & $(39)$ & 75 & (34) & 0 & \\
\hline Both rows & 0 & & 32 & (19) & 112 & $(51)$ & 7 & $(100)$ \\
\hline Number of flaps with necrosis & 6 & $(7)$ & 12 & $(7)$ & 22 & $(10)$ & 0 & \\
\hline
\end{tabular}

aFisher's exact test $p<0.001$.

possible small and asymptomatic necrosis. This surely affects the rate of necrosis found in our study compared with other studies.

In accordance with the findings of previous studies, ${ }^{20}$ we did not find any significant differences between IMV and TDV regarding postoperative re-anastomosis or flap survival. In our clinic, IMV is the first choice for anastomosis. Most of our DIEPs are delayed, and although IMV perforators are very popular and have been proposed for primary recipient vessels since $2007,{ }^{17}$ we do not usually have the chance to use them, as they are not preserved in primary operation. Indeed, according to the literature, IMV perforators are present in only $13 \%$ of delayed reconstructions. ${ }^{17}$ The anastomosis was mainly performed on TDV in primary reconstructions and in conversion cases. We had a $14 \%$ conversion rate from IMV to TDV, which is in line with earlier studies that reported conversion rates of 2 to $20 \% .^{19,20}$ The highest rate has been reported in previously radiated patients. ${ }^{20}$ In our study, over half of the patients with a conversion had undergone previous radiation therapy, which may have had an impact on this finding. According to clinical records, however, the most common reason for conversion was inadequate IM veins. A variation in IM vein size between the left and right sides has been described in the literature,

Table 3 The association between the incidence of necrosis and variables of interest ( $n=471$ deep inferior epigastric perforator)

\begin{tabular}{|c|c|c|c|c|c|c|}
\hline & \multirow[b]{2}{*}{$n$} & \multicolumn{5}{|c|}{ Flaps with necrosis $(n=40 ; 8.5 \%)$} \\
\hline & & $n$ & (\%) & OR & $(95 \%)$ & $p$-Value \\
\hline Age & 471 & 40 & (9) & 0.97 & $(0.93-1.004)$ & 0.080 \\
\hline \multicolumn{7}{|l|}{$\mathrm{BMI}$} \\
\hline$<30$ & 395 & 29 & (7) & 1.00 & & \\
\hline$\geq 30$ & 76 & 11 & (14) & 2.26 & $(1.05-4.87)$ & 0.038 \\
\hline \multicolumn{7}{|c|}{ History of abdominal operations } \\
\hline No & 344 & 32 & (9) & 1.00 & & \\
\hline Yes & 127 & 8 & (6) & 0.68 & $(0.30-1.54)$ & 0.352 \\
\hline \multicolumn{7}{|c|}{ Number of the perforator } \\
\hline $1-2$ & 246 & 18 & (7) & 1.00 & & \\
\hline$\geq 3$ & 225 & 22 & $(10)$ & 1.48 & $(0.72-3.05)$ & 0.284 \\
\hline \multicolumn{7}{|c|}{ Perforator location } \\
\hline Medial & 157 & 8 & (5) & 1.00 & & \\
\hline Lateral & 163 & 23 & (14) & 2.82 & $(1.19-6.70)$ & 0.019 \\
\hline Both & 151 & 9 & (6) & 0.98 & $(0.34-2.86)$ & 0.974 \\
\hline \multicolumn{7}{|l|}{ Radiation } \\
\hline No & 201 & 15 & (7) & 1.00 & & \\
\hline Yes & 270 & 25 & (9) & 1.26 & $(0.63-2.50)$ & 0.512 \\
\hline
\end{tabular}

Abbreviation: OR, odds ratio.

Note: Multivariable-adjusted logistic regression results were shown by odds ratios with $95 \%$ confidence intervals. 
Necrosis or Flap Loss After DIEP Reconstruction Palve et al. e25

Table 4 Analysis of recipient vessels and patient characteristics in whole study group $(n=471)$ and detailed analysis of reanastomosis

\begin{tabular}{|c|c|c|c|c|c|}
\hline \multirow[b]{2}{*}{ Number of DIEPs, $n(\%)$} & \multicolumn{2}{|l|}{ IMV } & \multicolumn{2}{|l|}{ TDV } & \multirow[t]{2}{*}{$p$-Value } \\
\hline & 287 & $(61)$ & 184 & (39) & \\
\hline \multicolumn{6}{|l|}{ Patient characteristics } \\
\hline Age of patient (y), median (range) & 52 & $(19-71)$ & 52 & $(29-71)$ & 0.740 \\
\hline BMI $\left(\mathrm{kg} / \mathrm{m}^{2}\right)$, median (IQR) & 26.4 & $(23.6-28.8)$ & 26.5 & $(24.6-29.0)$ & 0.209 \\
\hline $\mathrm{BMI} \geq 30, n(\%)$ & 44 & $(15)$ & 32 & $(17)$ & 0.553 \\
\hline Cardiovascular disease & 40 & (14) & 29 & (16) & 0.585 \\
\hline Radiotherapy, $n$ (\%) & 166 & $(58)$ & 104 & (57) & 0.778 \\
\hline Axillary clearance, $n$ (\%) & 139 & $(48)$ & 107 & $(58)$ & 0.039 \\
\hline Problems in vessel preparation & 11 & $(4)$ & 53 & (29) & $<0.001$ \\
\hline Re-anastomosis, $n(\%)$ & 12 & $(4)$ & 10 & (5) & 0.529 \\
\hline Thrombosis, $n(\%)$ & & & & & 1.000 \\
\hline Venous & 9 & $(75)$ & 7 & $(70)$ & \\
\hline Arterial & 3 & $(25)$ & 3 & $(30)$ & \\
\hline Flap survived, $n$ (\%) & 8 & $(67)$ & 8 & $(80)$ & 0.646 \\
\hline Postoperative day of problem, median (IQR; range) & 1 & $(0-3 ; 0-8)$ & 1 & $(0-5.5 ; 0-8)$ & 0.722 \\
\hline
\end{tabular}

Abbreviations: IMV, internal mammary vessels; IQR, interquartile range; TDV, thoracodorsal vessels.

Note: Differences between recipient vessels were analyzed by using Pearson's Chi-square test, Fisher's exact test, or Mann-Whitney test.

which is not described for TDV. ${ }^{18}$ In some reports, up to $20 \%$ of IM veins are reported to be of inadequate size (especially on the left side). ${ }^{18}$ This supports our findings that problems with veins were observed more commonly in left-sided vessels.

The rate of problems in the preparation of vessels was higher in TDV than IMV. According to clinical records, this higher rate was mainly due to postoperative scars in the axilla. Over half of these patients had a history of axillary clearance and radiation therapy. In these TDV problem cases, the preparation of vessels more proximally solved the problem, and no conversion was required. In our clinic, the maximum pedicle length is harvested in the DIEP flaps, allowing for the use of axillary vessels as alternative recipient vessels if problems with IMV occur. In the literature, thoracodorsal vessels are unusable in 7 to $15 \%$ of reconstructions in irradiated patients. ${ }^{20}$ These rates are, however, surgeon dependent. Thus, in some studies, vessels might be rejected without any exploration due to a preoperative hunch that there might be too much scar tissue in the axilla. $^{20}$

The perfusion of DIEP flaps can also be affected by venous draining capacity. Indeed, venous congestion is a common cause of flap failure. In previous studies, the rate of venous congestion has been reported to be up to $8 \%{ }^{3}$ In this present study, the problem of venous draining was also a more common reason for re-anastomosis than arterial thrombosis. Usually, the venous drainage of the DIEP flap is via the deep inferior epigastric vein. Sometimes, however, the dominant drainage is superficially via the superficial inferior epigastric vein (SIEV). ${ }^{3,22}$ Therefore, we always preserve the SIEV to salvage the vessel, as suggested by Blondeel et al. ${ }^{23}$ It has been concluded that drainage of the flap may be optimized through the deep drainage system if the venous perforators are of a sufficient size. Additional perforators may also decrease resistance and enhance drainage. If the venous perforators are too small or the connections between the deep and superficial systems are insufficient, salvage procedures may be necessary. ${ }^{24}$ If the congestion is identified intraoperatively, an additional venous anastomosis can prevent flap failure. $^{22}$ In this study, a second vein anastomosis was performed intraoperatively in $4 \%$ of flaps, which is comparable to the findings of previous studies. ${ }^{16}$ We have performed most of the second vein anastomosis from the SIEV to axillary vessels. However, in some cases, the anastomosis was performed to a branch of the DIEV in the "dualplane" style described earlier by Sbitany et al. ${ }^{25}$ In cases with co-dominant superficial and deep venous systems, a second anastomosis from other deep vena comitans to the retrograde internal mammary vein might also be a good option. ${ }^{26}$

We found that harvested perforators did not have an impact on the flap loss rate, but there was an increase in the flap necrosis requiring re-surgery in those flaps with three to five perforators compared with those flaps with one or two perforators. Controversial results have been published regarding the number of perforators and flap necrosis. A prior study by Gill et $\mathrm{al}^{16}$ demonstrated a significant increase in flap complications when more than one perforator was harvested. Conversely, Baumann et $\mathrm{al}^{15}$ found that flaps with one to two perforators had a higher incidence of fat necrosis than those with multiple perforators. A similar finding was reported by Grover et $\mathrm{al}^{14}$ for flaps with a single perforator. Our study is more in agreement with the findings of the study by Gill et al, ${ }^{16}$ who also concluded that the blood 
supply to the flap is poorer when using multiple suboptimal perforators compared with one or two strong ones. In our study, DIEP flaps most commonly incorporated three to five perforators, which is in line with previous studies. ${ }^{3,15}$ The number of perforators in our study was counted intraoperatively, and the selection of the perforators was planned preoperatively and based on angiographic imaging. Flaps were harvested with multiple perforators when there was a lack of large perforators.

In this study, one independent factor associated with flap necrosis was $\mathrm{BMI}>30$. According to earlier studies, the impact of BMI on the occurrence of fat necrosis in abdominally based flaps is not clear. Both a higher incidence of fat necrosis related to patient weight and no difference compared with normal-weight patients have been reported. ${ }^{27}$ It is also important to consider the size of the flap because obese patients have more commonly bigger flaps. On the other hand, the perforators of obese patients are also often larger in caliber and can supply the larger flap. ${ }^{16}$ It has, however, been concluded that as flap weight increases from 500 to $1,500 \mathrm{~g}$, the probability of fat necrosis also increases from 11.25 to $25 \%{ }^{5}$ In this study, we were unable to acquire information on flap weight because of the retrospective nature of the study. It might therefore be more meaningful to adjust for flap weight instead of patient BMI as a variable in future studies.

In previous studies, it has been suggested that successful free tissue transfer can be achieved in patients who smoke despite the higher rates of partial flap losses and woundhealing disturbances. ${ }^{28}$ In our study, however, only $8 \%$ of patients were smokers. This was a very small group of patients, and we did not find any statistical difference in complications between smokers and nonsmokers.

In our study, $27 \%$ of patients had lower abdominal scars due to previous surgery. An earlier study by Daly et al suggested that patients who have undergone prior abdominal surgery are at an increased risk for donor-site wound breakdown and fat necrosis of the reconstructed breast. ${ }^{29}$ However, prior lower abdominal surgery/scars were not associated with increased flap necrosis in our findings. It should be noted, however, that the risk for donor-site wound breakdown was outside the scope of this study.

The other independent factor associated with flap necrosis was perforator/s located on the lateral row. Mixed results have also been reported regarding this subject. According to the "perforasome" concept, medial row perforators have a larger and more centralized perfusion area, which makes them more suitable for large reconstructions, whereas lateral row perforators have a smaller perfusion zone that does not cross the midline. ${ }^{3,11}$ Other studies have suggested that despite the large calibers of medial row perforators, they have a higher incidence of necrosis because a fewer number of perforators are chosen in such cases. ${ }^{3}$ In fact, if medial row perforators are selected, the majority of the flaps are based on a single perforator compared with flaps with lateral row perforators, where only half are based on a single perforator. ${ }^{3}$ In our study, $70 \%$ of the flaps with a single perforator were also based on a medial row perforator, which is in line with the findings of previous studies. Some studies have also reported that using larger caliber perforators and perforators from the lateral row alone can decrease necrosis. ${ }^{5}$ The latter is the direct opposite of the finding in our study. The findings are, however, different with regard to hemiflaps because the occurrence of necrosis has been found to be significantly higher in flaps based solely on the medial row versus lateral row perforators. It has also been suggested that the addition of a lateral row perforator to a dominant medial row perforator may decrease the risk for necrosis in hemiflaps. ${ }^{30}$

This study has some limitations, including its retrospective nature. All the information about the perforators harvested, recipient vessels used, and complications was recorded from clinical files. We did not have any information about asymptomatic fat necrosis because we do not routinely use ultrasound to identify possible small and asymptomatic necrosis, which is a clear weakness of this study. The study was not randomized, as it would not have been ethically possible to randomly decide to perform a single or multiple perforator flap. The perforator/s diameter was not measured. However, in our clinic, flaps are harvested with multiple perforators when larger perforators are not available.

\section{Conclusion}

We conclude that the occurrence of flap necrosis requiring re-operation may be higher in DIEP flaps with more than two perforators or perforator/s located on the lateral row and in obese patients. Neither the recipient vessels used nor the number of perforators harvested had any impact on the flap survival rate.

\section{Funding}

This study received its financial support from Tampere University Hospital support foundation.

Conflict of Interest

None declared.

\section{References}

1 Lee KT, Mun GH. Perfusion of the DIEP flaps: a systematic review with meta-analysis. Microsurgery 2018;38(01):98-108

2 Mijuskovic B, Tremp M, Heimer MM, et al. Color Doppler ultrasound and computed tomographic angiography for perforator mapping in DIEP flap breast reconstruction revisited: a cohort study. J Plast Reconstr Aesthet Surg 2019;72(10):1632-1639

3 Bhullar H, Hunter-Smith DJ, Rozen WM. Fat necrosis after DIEP flap breast reconstruction: a review of perfusion-related causes. Aesthetic Plast Surg 2020;44(05):1454-1461

4 Hummelink S, Hoogeveen YL, Schultze Kool LJ, Ulrich DJO. A new and innovative method of preoperatively planning and projecting vascular anatomy in DIEP flap breast reconstruction: a randomized controlled trial. Plast Reconstr Surg 2019;143(06): 1151e-1158e

5 Hembd A, Teotia SS, Zhu H, Haddock NT. Optimizing perforator selection: a multivariable analysis of predictors for fat necrosis and abdominal morbidity in DIEP flap breast reconstruction. Plast Reconstr Surg 2018;142(03):583-592

6 Rose JF, Zavlin D, Garrett AE, Chegireddy V, Ellsworth WA IV. Evaluation of the single medial circummuscular perforator DIEP 
flap: outcomes and comparison to traditional transmuscular single perforator flap. Microsurgery 2018;38(05):479-488

7 Scheflan M, Dinner MI. The transverse abdominal island flap: part I. Indications, contraindications, results, and complications. Ann Plast Surg 1983;10(01):24-35

8 Scheflan M, Dinner MI. The transverse abdominal island flap: part II. Surgical technique. Ann Plast Surg 1983;10(02):120-129

9 Hartrampf CR, Scheflan M, Black PW. Breast reconstruction with a transverse abdominal island flap. Plast Reconstr Surg 1982;69 (02):216-225

10 Holm C, Mayr M, Höfter E, Ninkovic M. Perfusion zones of the DIEP flap revisited: a clinical study. Plast Reconstr Surg 2006;117(01): 37-43

11 Saint-Cyr M, Wong C, Schaverien M, Mojallal A, Rohrich RJ. The perforasome theory: vascular anatomy and clinical implications. Plast Reconstr Surg 2009;124(05):1529-1544

12 Wade RG, Watford J, Wormald JCR, Bramhall RJ, Figus A. Perforator mapping reduces the operative time of DIEP flap breast reconstruction: a systematic review and meta-analysis of preoperative ultrasound, computed tomography and magnetic resonance angiography. J Plast Reconstr Aesthet Surg 2018;71(04):468-477

13 Rozen WM, Bhullar HK, Hunter-Smith D. How to assess a CTA of the abdomen to plan an autologous breast reconstruction. Gland Surg 2019;8(Suppl 4):S291-S296

14 Grover R, Nelson JA, Fischer JP, Kovach SJ, Serletti JM, Wu LC. The impact of perforator number on deep inferior epigastric perforator flap breast reconstruction. Arch Plast Surg 2014;41(01):63-70

15 Baumann DP, Lin HY, Chevray PM. Perforator number predicts fat necrosis in a prospective analysis of breast reconstruction with free TRAM, DIEP, and SIEA flaps. Plast Reconstr Surg 2010;125 (05):1335-1341

16 Gill PS, Hunt JP, Guerra AB, et al. A 10-year retrospective review of 758 DIEP flaps for breast reconstruction. Plast Reconstr Surg 2004;113(04):1153-1160

17 Santanelli Di Pompeo F, Longo B, Sorotos M, Pagnoni M, Laporta R. The axillary versus internal mammary recipient vessel sites for breast reconstruction with diep flaps: a retrospective study of 256 consecutive cases. Microsurgery 2015;35(01):34-38

18 Banwell M, Trotter D, Ramakrishnan V. The thoracodorsal artery and vein as recipient vessels for microsurgical breast reconstruction. Ann Plast Surg 2012;68(05):542-543

19 Leppard W, Pomposelli T, Chang EI, Suliman A, Herrera F. Internal mammary usability as recipient vessels in DIEP breast recon- struction in the setting of previous radiation. J Plast Reconstr Aesthet Surg 2018;71(08):1123-1128

20 Temple CL, Strom EA, Youssef A, Langstein HN. Choice of recipient vessels in delayed TRAM flap breast reconstruction after radiotherapy. Plast Reconstr Surg 2005;115(01):105-113

21 Opsomer D, D’Arpa S, Benmeridja L, Stillaert F, Noel W, Van Landuyt K. Bilateral DIEP flap breast reconstruction to a single set of internal mammary vessels: technique, safety, and outcomes after 250 flaps. Plast Reconstr Surg 2019;144(04):554e-564e

22 La Padula S, Hersant B, Noel W, et al. Use of the retrograde limb of the internal mammary vein to avoid venous congestion in DIEP flap breast reconstruction: further evidences of a reliable and time-sparing procedure. Microsurgery 2016;36(06): 447-452

23 Blondeel PN, Arnstein M, Verstraete K, et al. Venous congestion and blood flow in free transverse rectus abdominis myocutaneous and deep inferior epigastric perforator flaps. Plast Reconstr Surg 2000;106(06):1295-1299

24 Karadsheh MJ, Shafqat MS, Krupp JC, Weiss ES, Patel SA. A theoretical model describing the dynamics of venous flow in the DIEP flap. J Reconstr Microsurg 2019;35(09):688-694

25 Sbitany H, Lentz R, Piper M. The "dual-plane" DIEP flap: measuring the effects of superficial arterial and venous flow augmentation on clinical outcomes. J Reconstr Microsurg 2019;35(06): 411-416

26 Kerr-Valentic MA, Gottlieb LJ, Agarwal JP. The retrograde limb of the internal mammary vein: an additional outflow option in DIEP flap breast reconstruction. Plast Reconstr Surg 2009;124(03): 717-721

27 Bozikov K, Arnez T, Hertl K, Arnez ZM. Fat necrosis in free DIEAP flaps: incidence, risk, and predictor factors. Ann Plast Surg 2009; 63(02):138-142

28 Prantl L, Moellhoff N, Fritschen UV, et al. Impact of smoking status in free deep inferior epigastric artery perforator flap breast reconstruction: a multicenter study. J Reconstr Microsurg 2020; 36(09):694-702

29 Daly LT, Doval AF, Lin SJ, Tobias A, Lee BT, Dowlatshahi AS. Role of CTA in women with abdominal scars undergoing DIEP breast reconstruction: review of 1,187 flaps. J Reconstr Microsurg 2020; 36(04):294-300

30 Kamali P, Lee M, Becherer BE, et al. Medial row perforators are associated with higher rates of fat necrosis in bilateral DIEP flap breast reconstruction. Plast Reconstr Surg 2017;140(01):19-24 